\title{
Tunable light trapping for solar cells using localized surface plasmons
}

\author{
F. J. Beck, ${ }^{1, a)}$ A. Polman, ${ }^{2}$ and K. R. Catchpole ${ }^{1,2}$ \\ ${ }^{1}$ Center for Sustainable Energy Systems, College of Engineering and Computer Science, Australian National \\ University, Canberra ACT 0200, Australia \\ ${ }^{2}$ Center for Nanophotonics, FOM Institute AMOLF, Kruislaan 407, 1098 SJ Amsterdam, The Netherlands
}

(Received 15 December 2008; accepted 30 April 2009; published online 8 June 2009)

\begin{abstract}
Effective light management is imperative in maintaining high efficiencies as photovoltaic devices become thinner. We demonstrate a simple and effective method of enhancing light trapping in solar cells with thin absorber layers by tuning localized surface plasmons in arrays of Ag nanoparticles. By redshifting the surface plasmon resonances by up to $200 \mathrm{~nm}$, through the modification of the local dielectric environment of the particles, we can increase the optical absorption in an underlying $\mathrm{Si}$ wafer fivefold at a wavelength of $1100 \mathrm{~nm}$ and enhance the external quantum efficiency of thin $\mathrm{Si}$ solar cells by a factor of 2.3 at this wavelength where transmission losses are prevalent. Additionally, by locating the nanoparticles on the rear of the solar cells, we can avoid absorption losses below the resonance wavelength due to interference effects, while still allowing long wavelength light to be coupled into the cell. Results from numerical simulations support the experimental findings and show that the fraction of light backscattered into the cell by nanoparticles located on the rear is comparable to the forward scattering effects of particles on the front. Using nanoparticle self-assembly methods and dielectrics commonly used in photovoltaic fabrication this technology is relevant for application to large-scale photovoltaic devices. () 2009 American Institute of Physics. [DOI: 10.1063/1.3140609]
\end{abstract}

\section{INTRODUCTION}

Photovoltaics is emerging as an important technology for the future of energy production. To fully realize this potential it will be necessary to reduce the manufacturing costs of photovoltaic cells while maintaining high working efficiencies. Thin film solar cells offer the advantage of reduced material costs but, as absorber layers become thinner, transmission losses increase and reduce cell performance. Light trapping, which can be used to confine light within the active layer to promote absorption, is becoming even more critical to achieving high efficiencies. Surface texturing has been successfully employed to enhance light absorption in wafer based Si cells ${ }^{1}$ but cannot be applied to thin film cells as textured features, up to $10 \mu \mathrm{m}$ in size, are much larger than the typical thin film thickness of 1-2 $\mu \mathrm{m}$. Wavelength scale surface texturing has also been shown to increase short circuit current by directing light into the cell at high propagation angles, ${ }^{2}$ but can also increase surface recombination and make the fabrication of good quality semiconductor layers more difficult. A novel approach that has emerged is the use of scattering by metal nanoparticles for light trapping in solar cells $;^{3-7}$ one of the many applications to have come out of the very active field of Plasmonics, a good review of which can be found in Ref. 8. Pillai et $\mathrm{al}^{7}{ }^{7}$ demonstrated an increase in photocurrent of up to 16-fold at long wavelengths due to the presence of Ag nanoparticles and showed that nanoparticle arrays can be used to enhance the performance of both thin film and wafer based $\mathrm{Si}$ solar cells. In that work, the $\mathrm{Ag}$ nanoparticles had a surface plasmon resonance at a wavelength of around $500 \mathrm{~nm}$. To optimize cell performance, light

${ }^{a)}$ Electronic mail: fiona.beck@anu.edu.au. trapping should be maximized for spectral regions where it is most needed. Lim et al. ${ }^{9}$ recently reported experimental and modeling results showing both enhancement and suppression of absorption as a function of wavelength due to the presence of nanoparticles. Clearly, greater control over the response of the nanoparticle films to different wavelengths is needed to fully utilize this effect for light trapping in solar cells.

In this study the ability to tune the surface plasmon resonance of metal nanoparticles is exploited for the design of light trapping layers that target the long wavelength regions where transmission losses are most significant for thin $\mathrm{Si}$ solar cells. Additionally, by changing the geometry of the system so that the nanoparticles are on the rear of the device, detrimental effects on the absorption are avoided. Ag nanoparticles with a radius of around $50 \mathrm{~nm}$ are chosen for their high scattering efficiency ${ }^{10}$ and the surface plasmon resonance wavelength $\left(\lambda_{\mathrm{SPR}}\right)$ is tuned to the desired spectral range by optimizing the local dielectric environment. ${ }^{11}$ The nanoparticles are formed directly on top of three different dielectrics commonly used in photovoltaic cell fabrication: silicon dioxide $\left(\mathrm{SiO}_{2}\right)$, silicon nitride $\left(\mathrm{Si}_{3} \mathrm{~N}_{4}\right)$, and titanium dioxide $\left(\mathrm{TiO}_{2}\right)$, with refractive indices of 1.5, 2.0, and 2.5, respectively. Nanoparticle fabrication employs a simple selfassembly method that is compatible with photovoltaic manufacturing procedures and suitable for large area devices such as solar cells. The $\lambda_{\text {SPR }}$ is redshifted by varying the underlying refractive index by up to $200 \mathrm{~nm}$, from a wavelength of $500 \mathrm{~nm}$ for particles on $\mathrm{SiO}_{2}$ to $700 \mathrm{~nm}$ for particles on $\mathrm{TiO}_{2}$. We study the effect of redshifted resonances on light trapping in Si using both optical absorption measurements on Si wafers and external quantum efficiency (EQE) measurements on crystalline Si solar cells. We show that light absorption, and subsequently photocurrent, is improved at long wave- 
lengths, by redshifting $\lambda_{\text {SPR }}$. Significantly these long wavelength enhancements are also evident with the nanoparticle arrays on the rear of the cells, providing light trapping at wavelengths where $\mathrm{Si}$ is a poor absorber while avoiding the reduction in photocurrent below resonance.

\section{EXPERIMENT}

Nanoparticle arrays for optical transmission and reflection measurements were fabricated on silica glass microscope slides as well as $200 \mu \mathrm{m}$, double side polished, $n$-type, $20 \Omega \mathrm{cm}$ Si wafers. Photocurrent measurements were performed on $100 \mu \mathrm{m}$ thick, bifacial crystalline Si solar cells, fabricated on $p$-type, $0.1 \Omega \mathrm{cm}$ wafers with $50 \Omega \mathrm{cm}$ phosphorous doped emitters.

As the solar cells were passivated with an oxide layer the Si wafer samples used for optical characterization had $15 \mathrm{~nm}$ of $\mathrm{SiO}_{2}$ grown in a dry oxygen environment for consistency. On top of the oxide layer, the Si samples received one of the following further dielectric layer depositions: $14 \mathrm{~nm}$ of thermally grown $\mathrm{SiO}_{2} ;(n=1.50), 17 \mathrm{~nm}$ of $\mathrm{Si}_{3} \mathrm{~N}_{4}(n=2.0)$ grown by low-pressure chemical vapor deposition (LPCVD), or 15 $\mathrm{nm}$ of $\mathrm{TiO}_{2}(n=2.5)$ grown by atmospheric-pressure chemical vapor deposition (APCVD). The glass slides used for optical characterization were either left bare or had either 17 $\mathrm{nm}$ of $\mathrm{Si}_{3} \mathrm{~N}_{4}$ or $15 \mathrm{~nm}$ of $\mathrm{TiO}_{2}$ deposited as described above.

Three types of bifacial solar cells were made, with oxide layers of $40 \mathrm{~nm}$ (sample A), $26 \mathrm{~nm}$ (sample B), and $26 \mathrm{~nm}$ (sample C). They were all coated with $20 \mathrm{~nm}$ of LPCVD $\mathrm{Si}_{3} \mathrm{~N}_{4}$. The $\mathrm{Si}_{3} \mathrm{~N}_{4}$ layer was then etched back to a final thickness of $3 \mathrm{~nm}$ (sample B) and $8 \mathrm{~nm}$ (sample C) and completely removed for sample A (residual thickness $<2 \mathrm{~nm}$ ). Sample $\mathrm{C}$ also received $15 \mathrm{~nm}$ of $\mathrm{TiO}_{2}$ by APCVD. All samples and cells were annealed at $400{ }^{\circ} \mathrm{C}$ for $30 \mathrm{~min}$ in forming gas before nanoparticle deposition.

Silver films with a thickness of $14 \mathrm{~nm}$ were deposited by thermal evaporation at a background pressure of less than $3 \mu$ Torr and annealed at $260{ }^{\circ} \mathrm{C}$ for $30 \mathrm{~min}$ in forming gas. When heated, the thin Ag films break up under surface tension to form isolated particles, the size and shape of which are sensitively dependent on the surface conditions of the underlying layer.

Scanning electron microscopy (SEM) images of the Ag layers after annealing were taken using a Zeiss UltraPLus field emission scanning electron microscope. The nanoparticle size, density, and shape were found to be dependent on the substrate due to the variation in surface conditions between sample types. Images were taken of Ag layers on $\mathrm{Si}$ wafers and the Si cells as well as for glass. Figure 1 shows micrographs of $\mathrm{Ag}$ nanoparticles on glass optical samples either left bare (a), or coated with LPCVD $\mathrm{Si}_{3} \mathrm{~N}_{4}$ (b), or APCVD $\mathrm{TiO}_{2}$ (c). The coalescing of nanoparticles is distinctly different on the three dielectrics. Nanoparticles formed on $\mathrm{SiO}_{2}$ had a Gaussian size distribution with an average radius of $68 \mathrm{~nm}$, a standard deviation of $43 \mathrm{~nm}$, and a surface coverage of $29 \%$. The structures formed on $\mathrm{Si}_{3} \mathrm{~N}_{4}$ and $\mathrm{TiO}_{2}$ had broader size distributions over a large number of sizes. For $\mathrm{Si}_{3} \mathrm{~N}_{4}$ the average radius was $62 \mathrm{~nm}$ with standard deviation of $64 \mathrm{~nm}$ and a surface coverage of $30 \%$. For

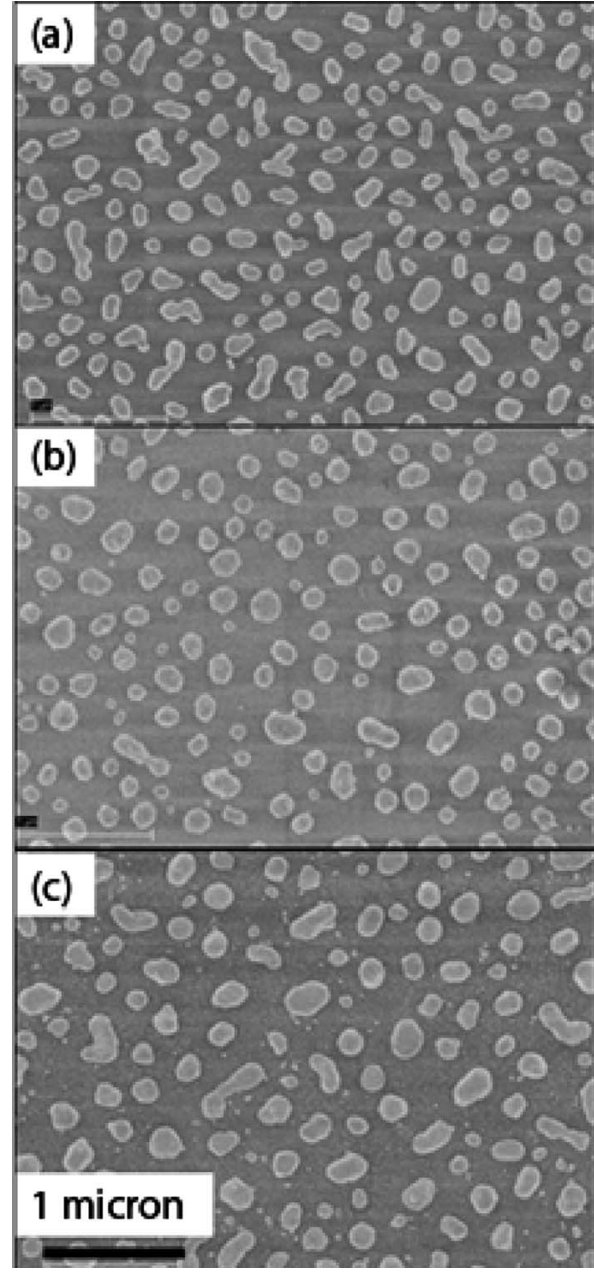

FIG. 1. SEM micrograph of Ag nanoparticles on bare glass slides (a) and glass coated with (b) $\mathrm{Si}_{3} \mathrm{~N}_{4}$ and (c) $\mathrm{TiO}_{2}$. Silver films with a thickness of 14 $\mathrm{nm}$ were deposited by thermal evaporation at a background pressure of less than $3 \mu$ Torr and annealed at $260{ }^{\circ} \mathrm{C}$ for $30 \mathrm{~min}$ in forming gas.

$\mathrm{TiO}_{2}$ the average radius was $64 \mathrm{~nm}$ with a standard deviation of $62 \mathrm{~nm}$ and $32 \%$ surface coverage. Shape anisotropy, defined as the average ratio of the minor and major axes of the nanoparticle cross section in the plane of the substrate, was 0.64 for $\mathrm{TiO}_{2}$ and 0.66 for $\mathrm{Si}_{3} \mathrm{~N}_{4}$, compared to 0.69 for $\mathrm{SiO}_{2}$ samples. No orientation dependences of the anisotropy were observed in the images of Fig. 1, as expected.

The nanoparticle films on glass and $\mathrm{Si}$ substrates were optically characterized using a dual-beam CARY 5000 Varian spectrophotometer with an integrating sphere attachment to measure total reflection and transmission. Extinction was calculated as 1 minus transmission, and absorption as 1 minus the sum of transmission and reflection.

The spectral response of the bifacial solar cells before and after nanoparticle deposition was determined using a halogen lamp source, chopped at a frequency of $70 \mathrm{~Hz}$, and filtered by a monochromator. The photocurrent at each wavelength, with a bandwidth of $5 \mathrm{~nm}$, was measured with a SR570 preamplifier and displayed as a voltage across a SR830 DSP lock-in amplifier. The EQE was then calculated from the known illumination intensity as the fraction of incident photons that are converted to electrical current. 


\section{NUMERICAL SIMULATIONS}

Numerical simulations were performed using the finitedifference time-domain (FDTD) solutions package from Lumerical software. A single Ag nanoparticle on a substrate was modeled using perfectly matched layer boundary conditions. Hemispheres were used to approximate the experimental nanoparticle shape. By simulating the single-particle case any interparticle interactions were ignored, allowing physical insights into the effects of the layered structure on the surface plasmon resonance and the absorption in the substrate. The extent to which this assumption is reasonable is discussed later by comparing the numerical and experimental results. A normally incident source was used to illuminate a semi-infinite substrate, comprised of either quartz or $\mathrm{Si}$, coated with thin dielectric layers of similar thicknesses as in the experimental cases. Radiation from the source was propagated from the air into to the substrate, corresponding to nanoparticles on the front of the samples, and from the substrate to the air, corresponding to nanoparticles on the rear. The dielectric functions were modeled using a Drude model for Ag and a Drude-Lorentz model for $\mathrm{Si}$. The three dielectrics under investigation were assumed to be nonabsorbing with a constant refractive index in the spectral region 500-1000 nm. The scattered and total (scattered plus incident) fields were calculated as a function of wavelength for each simulation.

To calculate the normalized absorption cross section $\left(Q_{\mathrm{abs}}\right)$ the Poynting vector of the total field was integrated over a box surrounding the nanoparticle and divided by the cross sectional area of the particle. The integrated Poynting vector of the scattered field was calculated separately in the air and in the substrate and the fraction of scattered light that was directed into the substrate $\left(f_{\text {sub }}\right)$ was evaluated as the scattered field in the substrate divided by the sum of the scattered field in the air and in the substrate. The normalized scattering cross section $\left(Q_{\text {scat }}\right)$ was determined in the same manner as $Q_{\mathrm{abs}}$ using the sum of the scattered fields. In the $\mathrm{Si}$ substrate the total transmitted field was calculated over a surface parallel to, and at a distance of $250 \mathrm{~nm}$, from the interface.

To determine the enhancement in the power transmitted into the $\mathrm{Si}$ due to the surface plasmon coupling, simulations were run with the nanoparticle present and compared to a reference simulation with no nanoparticle. The modeled enhancement was calculated as the ratio of the transmitted power in the Si with the nanoparticle present over the transmitted power in the reference case. The absolute magnitude of the modeled enhancements determined in this way depends on the area of the box over which total transmitted field is monitored. Box size was chosen to minimize simulation time while ensuring sufficient distance between monitors and interfaces for accurate simulation results.

\section{RESULTS AND DISCUSSION}

Figure 2 shows measured extinction spectra (solid lines) of $\mathrm{Ag}$ nanoparticles on glass substrates, (a) left bare, (b) coated with $\mathrm{Si}_{3} \mathrm{~N}_{4}$, and (c) $\mathrm{TiO}_{2}$. Broad resonances are observed with peak resonance wavelengths of $480 \mathrm{~nm}$ for $\mathrm{SiO}_{2}$,

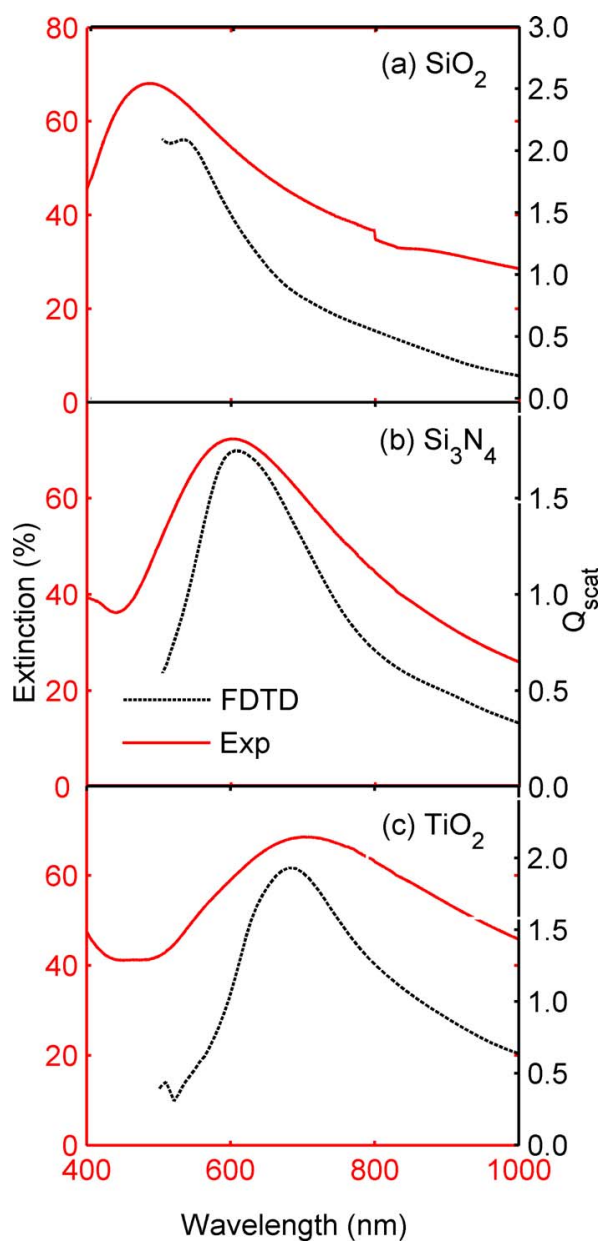

FIG. 2. (Color online) Experimental extinction spectra (solid lines, left axis) of Ag nanoparticle films on bare glass (a), glass coated with $\mathrm{Si}_{3} \mathrm{~N}_{4}$ (b), and $\mathrm{TiO}_{2}$ (c), along with modeled $Q_{\text {scat }}$ data calculated from FDTD simulations (dashed lines, right axis) of single Ag nanoparticles on glass substrates with dielectric layers similar to the three experimental cases. The scattering cross sections shown were calculated from the average of five different $Q_{\text {scat }}$ spectra of varying particle radii for each dielectric. The nanoparticle sizes were based on radii distributions observed in Fig. 1 with radii 40, 50, 55, 75, and $108 \mathrm{~nm}$ for $\mathrm{SiO}_{2}$ (a), 36, 50, 60, 76, and 116 for $\mathrm{Si}_{3} \mathrm{~N}_{4}$ (b), and 40, 50, 60, 82, and $118 \mathrm{~nm}$ for $\mathrm{TiO}_{2}(\mathrm{c})$.

$600 \mathrm{~nm}$ for $\mathrm{Si}_{3} \mathrm{~N}_{4}$, and $700 \mathrm{~nm}$ for $\mathrm{TiO}_{2}$. Clearly, the resonance redshifts with increasing refractive index of the underlying dielectric layer, demonstrating the tunability of the plasmon resonance in geometries that are typical for solar cell manufacturing.

Figure 2 also shows a calculation of the normalized $Q_{\text {scat }}$ (dashed lines). For each sample five characteristic nanoparticle radii were modeled and averaged using the measured size distributions on glass slides observed in Fig. 1 as a weighting factor. The overall trends in the experimental spectra are well represented by the weighted average. The modeled spectra are narrower than the broad experimentally observed ones due to the limited number of simulations performed for each sample. Additionally, interparticle coupling, which is not included in the simulations, may also contribute to the broadening of the resonance peaks in the experimental data.

Figure 3 illustrates the sensitivity of the scattering resonance wavelength of a $50 \mathrm{~nm}$ radius nanoparticle to the ge- 


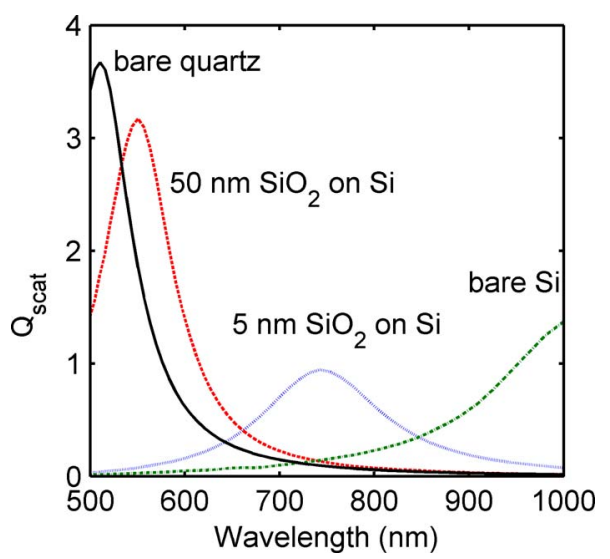

FIG. 3. (Color online) Modeled scattering cross sections of a single Ag nanoparticle with radius $50 \mathrm{~nm}$ on bare quartz (solid line), $50 \mathrm{~nm} \mathrm{SiO}$ on a Si substrate (dashed line), and $5 \mathrm{~nm} \mathrm{SiO}_{2}$ on a Si substrate (dotted line) and bare Si (dashed-dot line).

ometry of the dielectric layers and the substrate. Calculated scattering spectra are shown for $\mathrm{Ag}$ nanoparticles on bare quartz (solid line), bare Si (dashed line) and Si coated with 5 $\mathrm{nm} \mathrm{SiO}_{2}$ (dotted line), and $50 \mathrm{~nm} \mathrm{SiO}_{2}$ (dashed-dot line). Clearly, increased overlap of the particles near field with the high-index substrate causes a redshift in resonance. The resonance shifts to $550 \mathrm{~nm}$ for $50 \mathrm{~nm}$ of $\mathrm{SiO}_{2}$ and to $750 \mathrm{~nm}$ for $5 \mathrm{~nm}$ of $\mathrm{SiO}_{2}$. For $\mathrm{Ag}$ nanoparticles on bare $\mathrm{Si}$, the position of the scattering peak is strongly redshifted beyond $1000 \mathrm{~nm}$ and out of the wavelength range of the simulations. The strength of the resonance is also dependent on the distance of the nanoparticle from the substrate, suggesting that modification of the dielectric environment by the addition of highindex dielectrics is a more effective method of redshifting the $\lambda_{\mathrm{SPR}}$ for this application.

Figure 4 shows measured optical absorption spectra derived from a combination of reflection and transmission measurements of $200 \mu \mathrm{m}$ thick Si wafers with nanoparticles on the front (a) and the rear (b) of the samples (dashed lines). A reference measurement taken on a Si wafer with $15 \mathrm{~nm}$ of $\mathrm{SiO}_{2}$ thermally grown on the surface (solid lines) is included for comparison. By comparing measurements from both the front and rear configurations, scattering by $\mathrm{Ag}$ nanoparticles into the substrate for light incident from both the air and the $\mathrm{Si}$ can be studied. For light incident from the front of the samples [Fig. 4(a)], a large enhancement in absorption, by as much as $23 \%$, is observed for the $\mathrm{SiO}_{2}$ sample for wavelengths above $600 \mathrm{~nm}$. For the $\mathrm{Si}_{3} \mathrm{~N}_{4}$ layer an enhancement is seen only for wavelengths above $800 \mathrm{~nm}$; no enhancement is observed for the $\mathrm{TiO}_{2}$ below $1000 \mathrm{~nm}$. In all three geometries, an increase in absorption is observed at wavelengths below $500 \mathrm{~nm}$.

A local minimum in the absorption spectra in Fig. 4(a) is observed at $515 \mathrm{~nm}$ for $\mathrm{SiO}_{2}, 677 \mathrm{~nm}$ for $\mathrm{Si}_{3} \mathrm{~N}_{4}$, and $775 \mathrm{~nm}$ for $\mathrm{TiO}_{2}$. The position of the local minima and the spectral bandwidth over which absorption is reduced shows the same trend as the width and position of the scattering resonance peaks in Fig. 2, with $\mathrm{Si}_{3} \mathrm{~N}_{4}$ and $\mathrm{TiO}_{2}$ coated samples showing increasingly redshifted and wider resonances compared to the $\mathrm{SiO}_{2}$ case. The reduction in absorption around the plasmon resonance is attributed to interference between light

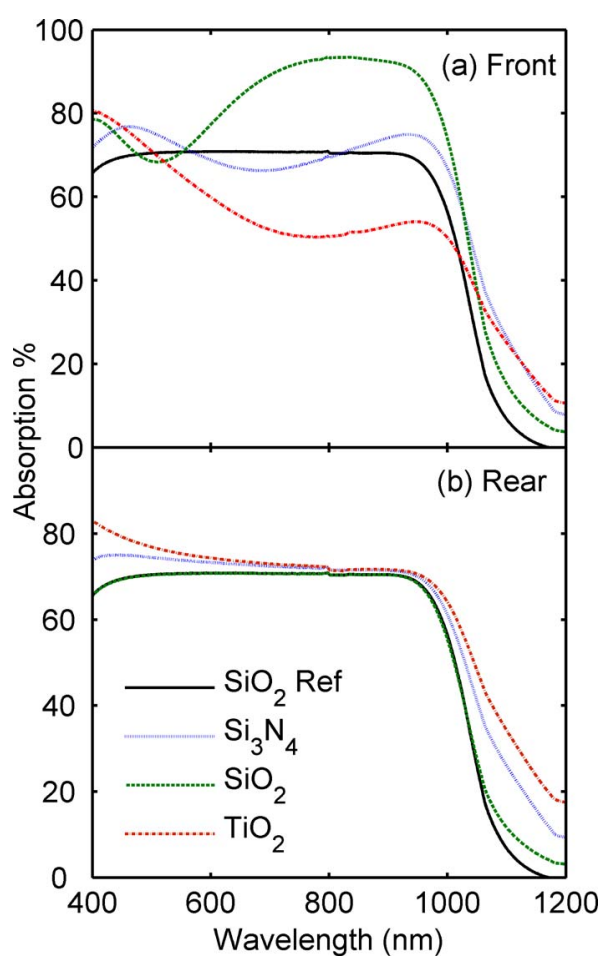

FIG. 4. (Color online) Measured absorption spectra derived from transmission and reflection measurements, on $200 \mu \mathrm{m}$ thick Si wafers. Ag nanoparticles films on layers of $\mathrm{SiO}_{2}, \mathrm{Si}_{3} \mathrm{~N}_{4}$, and $\mathrm{TiO}_{2}$ are plotted with illumination from the front (a) and from the rear (b). A reference measurement for a $\mathrm{Si}$ wafer with a $\mathrm{SiO}_{2}$ layer and no nanoparticles is shown in each graph.

that is transmitted at the Si interface and light that is scattered by the nanoparticles. For wavelengths below resonance, the scattered light is out of phase with the incident light, leading to destructive interference with the incoupled light. ${ }^{9}$ This effect is most clearly seen just below resonance where both the phase shift and the scattering cross section are significant. The small absorption enhancements observed for the shortest wavelengths are attributed to absorption by small $\mathrm{Ag}$ nanoparticles, which have a low scattering efficiency, and absorption in the different dielectric layers.

For light incident on the rear of the samples [Fig. 4(b)] a clear absorption enhancement is observed for all three dielectrics above $1000 \mathrm{~nm}$, while the major absorption dips around $\lambda_{\text {SPR }}$ seen in Fig. 4(a) are not observed. The latter is due to the fact that the Si wafer is strongly absorbing up to wavelengths of $900 \mathrm{~nm}$ (as seen from the reference case; solid line), so the nanoparticles are not expected to affect absorption in this spectral region with light incident from the rear. The slight increases in the absorption at short wavelengths in Fig. 4(b) are due to the different dielectric coatings on each wafer acting as short wavelength antireflection coatings. The absorption enhancement above $900 \mathrm{~nm}$ is largest for the $\mathrm{TiO}_{2}$ layer, consistent with the fact that the largest $\lambda_{\text {SPR }}$ redshift is observed for the highest-index dielectric (see Fig. 2). At a wavelength of $1100 \mathrm{~nm}, 7 \%$ absorption is observed for the reference case, $12 \%$ with nanoparticles on $\mathrm{SiO}_{2}$, and up to $26 \%$ and $34 \%$ for particles on $\mathrm{Si}_{3} \mathrm{~N}_{4}$ and $\mathrm{TiO}_{2}$, respectively. It is clear that enhanced absorption for wavelengths in the range $900-1200 \mathrm{~nm}$ occurs in both front and rear configurations. 


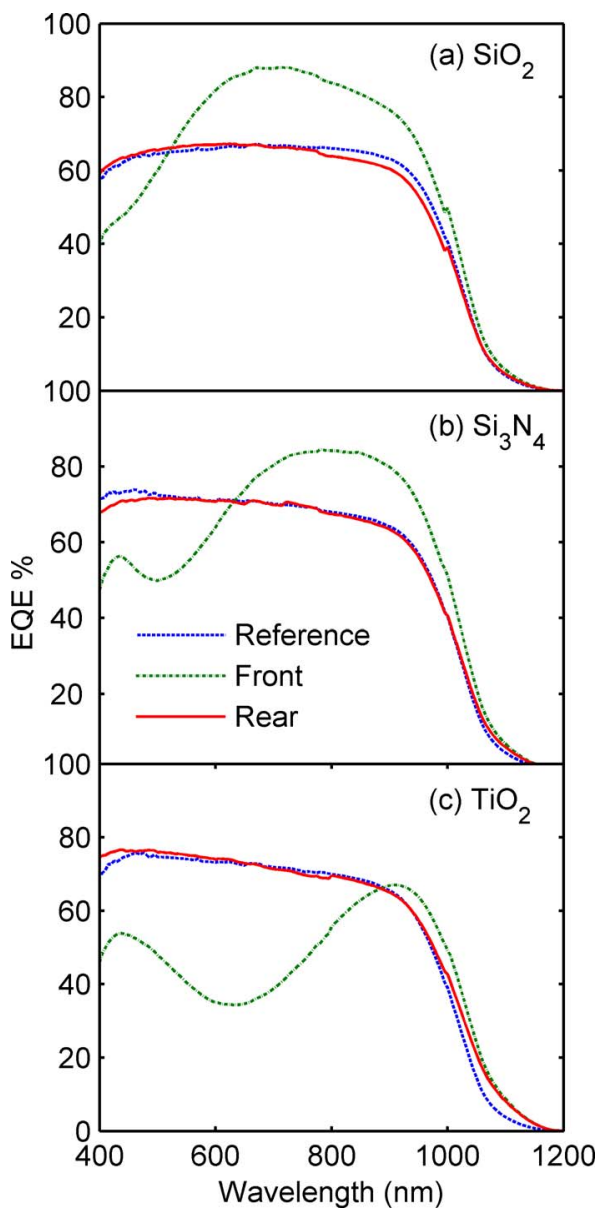

FIG. 5. (Color online) Measured EQE of $100 \mu \mathrm{m}$ thick, crystalline Si, bifacial solar cells, with nanoparticle arrays on the front (dashed-dot line) and the rear (solid line). Cells had a dielectric layer structure of $\mathrm{SiO}_{2}$ and $\mathrm{Si}_{3} \mathrm{~N}_{4}$ (etched off for sample A) with a top layer of (a) $\mathrm{SiO}_{2}$ on sample A, (b) $\mathrm{Si}_{3} \mathrm{~N}_{4}$ on sample $\mathrm{B}$, and (c) $\mathrm{TiO}_{2}$ on sample $\mathrm{C}$. Included is a reference measurement (dashed lines) without particles for each of the different dielectric configurations.

Figure 5 shows EQE spectra measured for bifacial $\mathrm{Si}$ solar cells with and without $\mathrm{Ag}$ nanoparticles and with different dielectric layer configurations. Measurements were taken with illumination from the front (dashed dot lines) and the rear (solid lines) side; reference measurements without nanoparticles (dashed lines) are also shown. The reference measurements for the three different dielectric layer configurations $(\mathrm{a}, \mathrm{b}$, and $\mathrm{c})$ show an increase in EQE for the higher index dielectrics as they act as antireflection coatings. A clear enhancement in $\mathrm{EQE}$ is observed for light incident from the front for sample A, with an uppermost layer of $\mathrm{SiO}_{2}$, and sample B with $\mathrm{Si}_{3} \mathrm{~N}_{4}$ on top. Interference effects around $\lambda_{\mathrm{SPR}}$ can be clearly seen. Small variations in the position of the local minima between Figs. 4 and 5 are ascribed to the differences in dielectric layer structure and $\mathrm{Ag}$ nanoparticle films produced by dissimilar surface conditions. This was confirmed using FDTD calculated scattering cross sections for layer geometries and nanoparticle sizes similar to samples A, B, and C showing $\lambda_{\mathrm{SPR}}$ at 550, 610, and $740 \mathrm{~nm}$, respectively. Figure 5 shows large absolute increases in $\mathrm{EQE}$ at wavelengths above resonance, of up to $24 \%$ at $650 \mathrm{~nm}$ for sample A (a) and $16 \%$ at $800 \mathrm{~nm}$ for sample B (b) when light

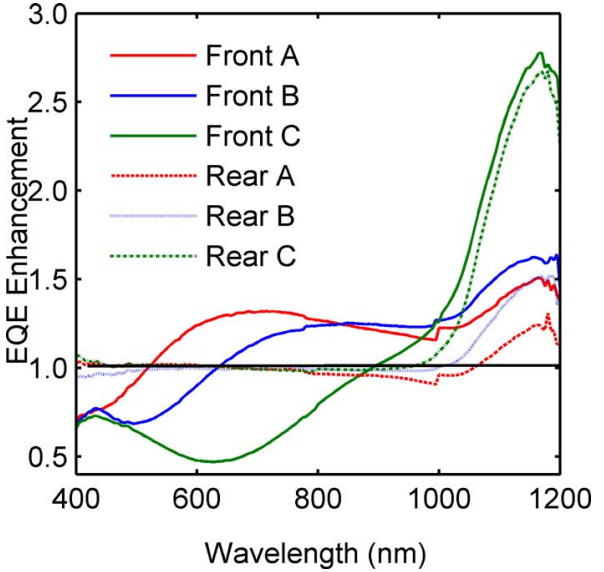

FIG. 6. (Color online) EQE enhancements for sample A, sample B, and sample $\mathrm{C}$ due to the presence of $\mathrm{Ag}$ nanoparticles, derived from the data from Fig. 5. Results are shown for illumination from the front (solid lines) and the rear (broken lines).

is incident from the front (dashed dot). For sample C (c), with particles on $\mathrm{TiO}_{2}$, enhancements are observed above $900 \mathrm{~nm}$.

Figure 6 plots the relative EQE enhancements derived from the data in Fig. 5.

At wavelengths between 1000 and $1100 \mathrm{~nm}$ all samples show large enhancements due to the nanoparticles. For sample $\mathrm{C}$ the EQE is enhanced by a factor of 2.3 when illuminated from the front and 2.1 when illuminated from the rear at $1100 \mathrm{~nm}$. Enhancements in this wavelength range are ascribed to light trapping provided by the nanoparticles scattering incident light at high angles into the substrate. This occurs whether light is incident on the front and on the rear, as seen in Fig. 6. We note that significant enhancements in absorption above $1100 \mathrm{~nm}$ seen in Fig. 4 do not correspond to an increase in EQE in Figs. 5 and 6. Due to the vicinity of the band edge at $1125 \mathrm{~nm}$ in crystalline $\mathrm{Si}$, absorption at wavelengths above this can be attributed to free carrier absorption, which will not contribute to the photocurrent. Absorption enhancements seen in Fig. 4 at short wavelengths are not evident in Figs. 5 and 6; indeed EQE is reduced in all cases around $400 \mathrm{~nm}$. This suggests that the additional absorption at this wavelength is due to absorption in small $\mathrm{Ag}$ nanoparticles, as suggested above, or is occurring very close to the Si surface and not in the active region of the cell.

In Fig. 7 the measured EQE enhancement data (solid lines) for illumination from the front from Fig. 6 are compared to the modeled enhancement of total power transmitted into the Si substrate due to the presence of a single nanoparticle (dashed lines). The power transmitted through the Si at a depth of $250 \mathrm{~nm}$ from the surface, as calculated in the FDTD simulations, is directly related to the photocurrent produced at low illumination intensities (for wavelengths in the range 500-1000 nm) assuming that all photons with sufficient energy are absorbed by the semiconductor and produce carriers, and that these carriers are collected in the external circuit. As the cells used in the experiment were well passivated and fabricated on high quality crystalline $\mathrm{Si}$, this is a valid assumption.

The overall shape of the modeled curves shows good 


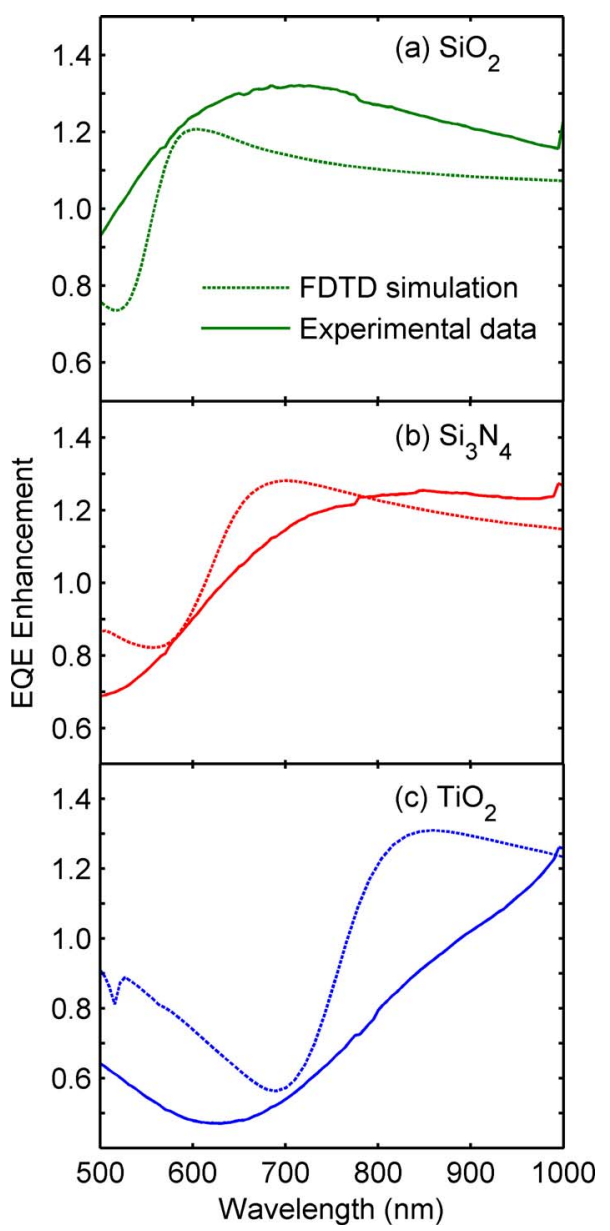

FIG. 7. (Color online) Modeled enhancements in transmitted power (dashed lines) due to the presence of a Ag nanoparticle with light incident from the front, compared to measured EQE enhancements (solid lines) for sample A (a), sample B (b), and sample C (c).

qualitative agreement with the experimental data for the three different dielectrics. The troughs observed experimentally are wider than those calculated by FDTD, which we attribute to the spread of particle radii and geometries in the Ag nanoparticle films, but the trends for both data sets are the same. The calculated curves show the same wavelength dependent enhancement and suppression as the measured data, with local minima at longer wavelengths as the refractive index is increased. Increases in experimentally measured EQE enhancement, at $1000 \mathrm{~nm}$ for $\mathrm{SiO}_{2}$ (a) and $\mathrm{Si}_{3} \mathrm{~N}_{4}$ (b) and above $900 \mathrm{~nm}$ for $\mathrm{TiO}_{2}$ (c), are not seen in modeled results as these are light trapping effects, which are not included in the simulation since the simulation assumes a semi-infinite substrate.

Figure 8 shows the fraction of the light scattered by the $\mathrm{Ag}$ nanoparticle that is scattered into the Si substrate. Although light-trapping effects cannot be directly modeled in the simulation due to the semi-infinite substrate used, the portion of scattered light that is directed into the substrate can give a good indication of how much light would be trapped in a real cell. Above wavelengths of $900 \mathrm{~nm}$ the fraction of light that is scattered into the $\mathrm{Si}$ is high for all three geometries, between $80 \%$ and $90 \%$, regardless of whether the cells are illuminated from the front or the rear.

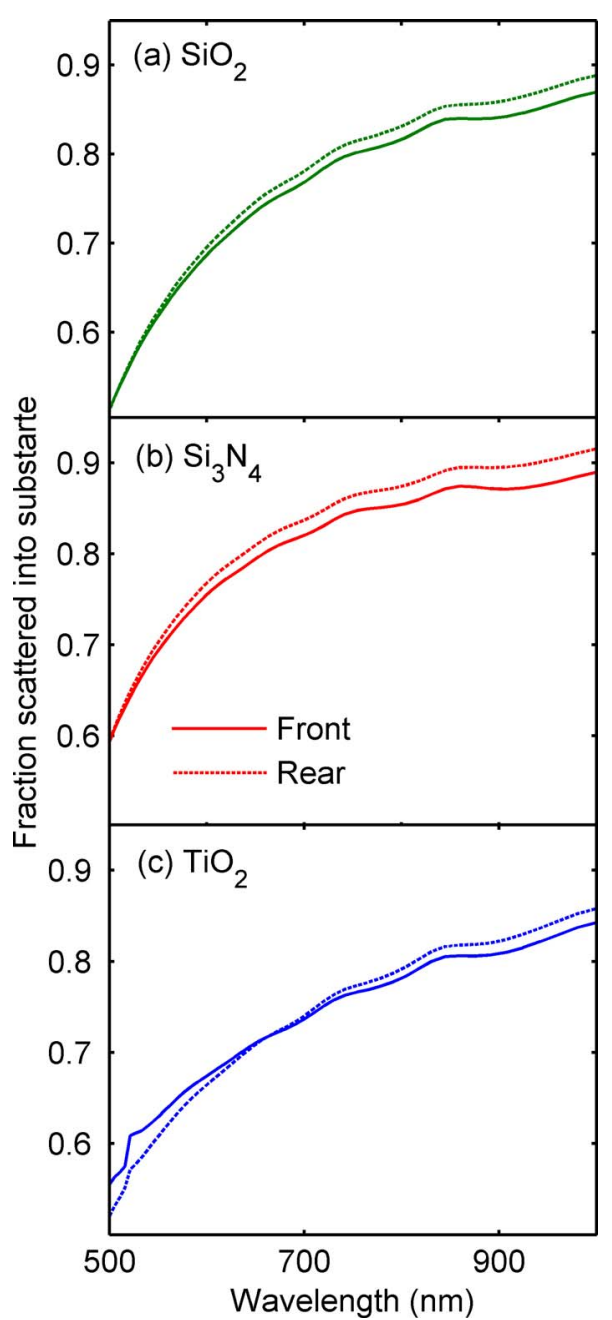

FIG. 8. (Color online) Modeled results for the fraction of scattered light scattered into the substrate due to the presence of a Ag nanoparticle. FDTD simulation calculations are shown for geometries similar to sample A (a), sample B (b), and sample C (c) for illumination from the front (solid lines) and the rear (dashed lines).

This is consistent with the particle response being dominated by the dipole mode, which in free space scatters symmetrically forward and backward. Higher order modes, which would have a significant contribution for larger particles, tend to be more forward scattering. ${ }^{12}$ This suggests that the EQE enhancements seen at long wavelengths under illuminated from both the front and the rear (Fig. 6) are indeed due to light trapping effects resulting from scattering by the nanoparticles.

\section{CONCLUSION}

Localized surface plasmon resonances in Ag nanoparticles can be tuned to longer wavelengths, where $\mathrm{Si}$ is a poor absorber of light, by varying the refractive index of underlying dielectric layers. For nanoparticles on the front, light trapping is observed for wavelengths above the surface plasmon resonance and a reduction in absorption is observed below resonance due to interference effects. Nanoparticles located on the rear of thin solar cells provide light trapping while avoiding the latter. This has a direct application as a simple and effective method of optimizing light trapping lay- 
ers for a variety of solar cells, especially those with thin absorber layers. Furthermore, light trapping for other absorber layers could be engineered using this method of targeting specific wavelength regions. Simulated enhancements clearly support experimental results, showing that by modifying the local dielectric environment the surface plasmon resonance can be optimized and the fraction of light absorbed in a solar cell can be increased in specific spectral regions.

${ }^{1}$ M. A. Green, Advances in Solar Energy 8, 231 (1993).

${ }^{2}$ R. H. Morf, H. Kiess, and C. Heine, in Diffractive Optics for Industrial and Commercial Applications, edited by J. Turunen and F. Wyrowski (Akademie Verlag, Berlin, 1997), p. 361.
${ }^{3}$ K. R. Catchpole and A. Polman, Appl. Phys. Lett. 93, 191113 (2008). ${ }^{4}$ D. M. Schaadt, B. Feng, and E. T. Yu, Appl. Phys. Lett. 86, 063106 (2005).

${ }^{5}$ B. P. Rand, P. Peumans, and S. R. Forrest, J. Appl. Phys. 96, 7519 (2004). ${ }^{6}$ D. Derkacs, S. H. Lim, P. Matheu, W. Mar, and E. T. Yu, Appl. Phys. Lett. 89, 093103 (2006).

${ }^{7}$ S. Pillai, K. R. Catchpole, T. Trupke, and M. A. Green, J. Appl. Phys. 101, 093105 (2007).

${ }^{8}$ S. A. Maier and H. A. Atwater, J. Appl. Phys. 98, 011101 (2005).

${ }^{9}$ S. H. Lim, W. Mar, P. Matheu, D. Derkacs, and E. T. Yu, J. Appl. Phys. 101, 104309 (2007).

${ }^{10}$ H. R. Stuart and D. G. Hall, Appl. Phys. Lett. 73, 3815 (1998).

${ }^{11}$ G. Xu, M. Tazawa, P. Jin, S. Nakao, and K. Yoshimura, Appl. Phys. Lett. 82, 3811 (2003).

${ }^{12}$ C. F. Bohren and D. R. Huffman, Absorption and Scattering of Light by Small Particles (WILEY-VCH Verlag GmbH \& Co. KGaA, Weinheim, 2004). 